\title{
Formation of Two-Carbon Sugar Fragment at an Early Stage of the Browning Reaction of Sugar with Amine
}

\author{
Tateki Hayashi and Mitsuo NAMIKI \\ Department of Food Science and Technology, Faculty of Agriculture. \\ Nagova University, Chikusa-ku, Nagova 464, Japan
}

Received March 26, 1980

\begin{abstract}
A product easily converted to glyoxal was found in an early stage of the reaction of sugar with amine in ethanol. Glyoxaldicyclohexylimine was isolated from the reaction mixture of D-glucose with cyclohexylamine. This finding suggested the formation of a similar type glyoxaldialkylimine in other reactions of sugar with amine. This two-carbon compound was assumed to be produced directly from sugar or glycosylamine, and a new pathway for sugar fragmentation was proposed.
\end{abstract}

It has been shown by earlier studies that the ESR signals observed at the earlier stages of the sugar-amine browning reactions are principally due to the formation of $N, N^{\prime}$ disubstituted pyrazine cation radicals. ${ }^{1)}$ This finding lead us to an assumption of the formation of a particular two-carbon fragment of sugar, which may be a carbonyl or an enaminol compound that acts as a precursor to the above radical species.

The generally accepted pathway of the browning reaction involves the formation of Schiff base, that of the Amadori rearrangement product, and the subsequent formation of osone. The cleavage of the sugar carbon chain at the early stage of reaction has so far received little attention, even though there has been sporadic report of the formation of twocarbon fragments, such as acetic acid. ${ }^{2)}$ The present study made it clear, mainly by means of isolation and identification of glyoxaldicyclohexylimine as one of the products of reaction of glucose with cyclohexylamine in $95 \%$ ethanol, that the two-carbon fragment is indeed formed at the early stage of the browning reaction.

\section{RESULTS AND DISCUSSION}

When the reaction mixtures of $\mathrm{D}$-glucose (or other hexose or pentose) with alkylamine are developed on silica gel TLC plate and sprayed with solution of 2,4-dinitrophenylhydrazine (2,4-DNP), a particular orange spot is always observed (Fig. 1). An additional spot is observed when the amine is ethyl- or $n$ butylamine but not when it is $t$-butyl- or cyclohexylamine. This has attracted our attention, and the nature of this substance was investigated. The substance was obtained in noncrystalline form by extraction of the corresponding preparative TLC band from the partly purified reaction mixture of glucose with $t$-butylamine in ethanol. GLC analysis of its reduced and acetylated product showed a main peak identical with that for diacetylated ethyleneglycol, but apparently differing from

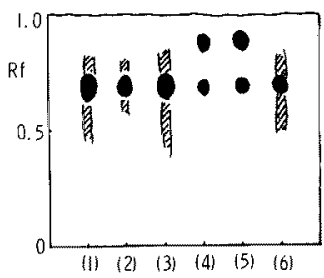

Fig. 1. TLC of the Reaction Mixtures of Sugars with Amines in Ethanol.

Mixtures of the materials ( $1 \mathrm{M}$ each) were prepared by refluxing for $40 \mathrm{~min}((1) \sim(3))$ or $10 \mathrm{~min}((4) \sim(6)) .2,4$ DNP-positive spots: (1) D-glucose and t-butylamine; (2) $\mathrm{D}$-fructose and $t$-butylamine; (3) $\mathrm{D}$-arabinose and $t$ butylamine: (4) D-glucose and $n$-butylamine; (5) Dglucose and cyclohexylamine. 


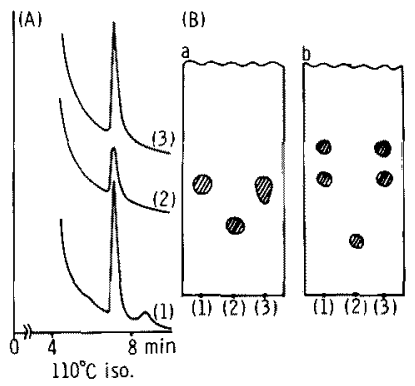

FIG. 2. GLC (A) and TLC (B) of the Isolated 2,4DNP-positive Substance.

TLC-a, 2,4-DNP-positive spots, developed chloroformethanol-water $(5: 2: 1)$. TLC-b, 2,4-DNP derivatives. On the details, see EXPERIMENTAL in the text. (1) isolated substance; (2) glycolaldehyde; (3) glyoxal.

acetylated glycerol (Fig. 2A). This suggested that the substance is glyoxal or glycolaldehyde, and the former was supported by TLC analyses of the substance itself and of its mixture with 2,4-DNP carried out in parallel with authentic glyoxal (Fig. 2B). Furthermore, phenylhydrazone of this substance could successfully be prepared, and its ${ }^{1} \mathrm{H}-\mathrm{NMR}$ and IR spectra agreed well with those of authentic glyoxalbishydrazone.

The identification of this substance as glyoxal, however, did not mean that it is the direct product of the reaction. This is because it was shown that a 2,4-DNP-positive product is certainly present in the reaction mixture, but this product is solvent-soluble and completely extracted with ethyl acetate, whereas glyoxal is not. It was further shown that the treatment of the reaction mixture by addition of acid or silica gel powder readily converted the product to glyoxal, which remained in aqueous layer for the extraction (Fig. 3). It therefore seemed reasonable to assume that glyoxal detected on TLC was the artifact of hydrolysis of the product in question in the mixture, effected by silica gel TLC procedure.

In order to isolate the unchanged 2,4-DNPpositive product from the reaction mixture, fractionation by solvent extraction was employed. Chloroform extraction of the reaction mixture of D-glucose with $n$ - or $t$-butylamine gave only noncrystalline product, but that

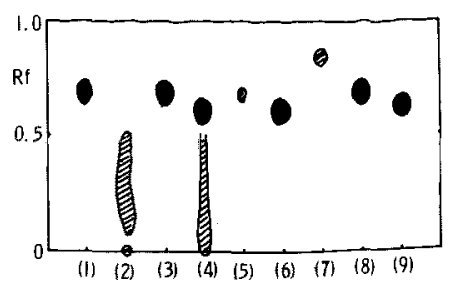

FIG. 3. TLC of the Reaction Mixture of D-Glucose with $t$-Butylamine before or after Acid- or Silica Geltreatment.

2,4-DNP-positive spots. On the details, see EXPERIMENTAL in the text. (1) reaction mixture; (2) water layer of the reaction mixture; (3) ethyl acetate layer of the reaction mixture; (4) water extract after silica geltreatment; (5) ethyl acetate extract after silica geltreatment; (6) water layer after acid-treatment; (7) ethyl acetate layer after acid-treatment; (8) glyoxal in ethanol; (9) glyoxal in water.

with cyclohexylamine yielded a colorless crystalline product. Mass spectrum of this product gave $\mathrm{M}^{+}$of 220 corresponding to the Schifftype condensation product of 2 molecules of cyclohexylamine with glyoxal. The significant peak at $m / e 177$ can be explained by the fragment

$$
\mathrm{CH}_{2}=\mathrm{CH}-\mathrm{CH}=\stackrel{+}{\mathrm{N}}=\mathrm{C} \mathrm{H}-\mathrm{CH}=\mathrm{N}-\mathrm{C}_{6} \mathrm{H}_{11}
$$

resulted from the ring fission of the cyclohexyl group, which is frequently seen for cyclohexylamine derivatives. ${ }^{31}{ }^{1} \mathrm{H}-\mathrm{NMR}$ of this product showed signals for two cyclohexylamino groups $(\delta 1.30 \sim 1.80$ and 3.60$)$ and a signal for two protons of the imine groups $(\delta 7.82)$. This spectrum as well as the IR spectrum (Fig. 4) agreed well with those of the authentic specimen of glyoxaldicyclohexylimine. The yield of this compound, as estimated by the procedure described in EXPERIMENTAL, was 12 or $74 \mathrm{mmol}$ from $1 \mathrm{~mol}$ glucose, when it was reacted with 1 or $5 \mathrm{~mol}$ cyclohexylamine, respectively.

When the isolated or authentic glyoxaldicyclohexylimine was developed on silica gel plate as was done for the reaction mixture, the only spot developed by 2,4-DNP was that of glyoxal, and heating the plate sprayed with sulfuric acid revealed no additional spot. It was also confirmed that the treatment of its 


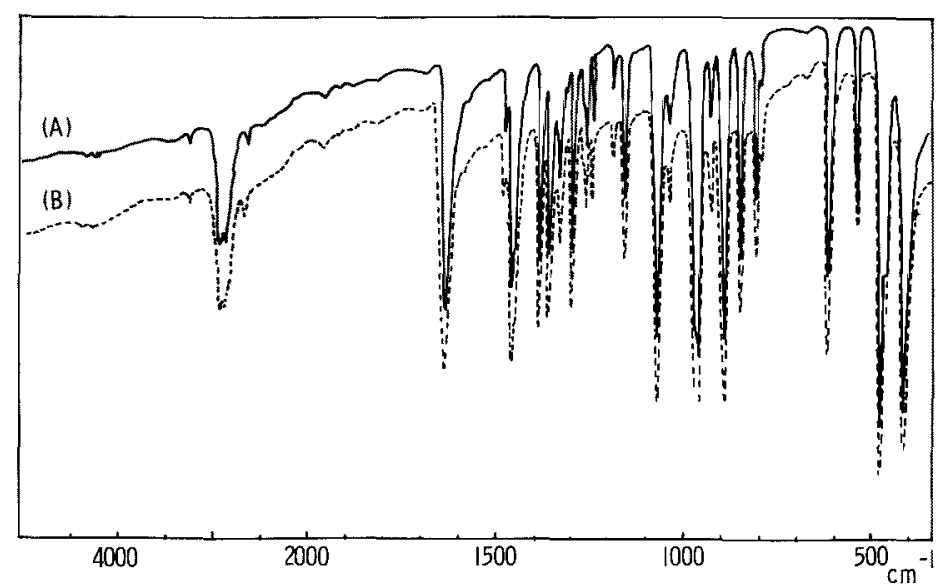

FIG. 4. IR Spectra of the Isolated Product (A) and Authentic Glyoxaldicyclohexylimine.

solution with acid or silica gel, carried out as was done before, readily hydrolyzed the imine to glyoxal.

These findings confirmed that glyoxaldicyclohexylimine is one of the products of the reaction of glucose with cyclohexylamine in ethanol. Although the formation of similar imines by the reaction of glucose with other alkylamines was not directly evidenced, the detection of glyoxal by silica gel TLC (Fig. 1) in all of these cases seems to justify the assumption that the two-carbon imines are as a rule among the products of this kind of reactions, under the conditions employed.

3-Deoxyglucosone is the recognized intermediate of the browning reaction of sugar with amines. ${ }^{4)}$ In order to clarify the relation of the formation of the two-carbon imines to that of 3-deoxyglucosone, the progress of the reaction of glucose with $t$-butylamine was followed by simultaneous analyses with respect to 3-deoxyglucosone, the two-carbon imine (as glyoxal), the free radical species (by ESR) and the degree of browning. The results are shown in Fig. 5. Analytical methods are described in EXPERIMENTAL. It is interesting to note that the two-carbon imine is formed at the very early stage of the reaction and its amount reaches maximum after $20 \mathrm{~min}$ of heating under reflux and then gradually decreases. This precedes either the initial formation of 3-deoxyglucosone, that of the

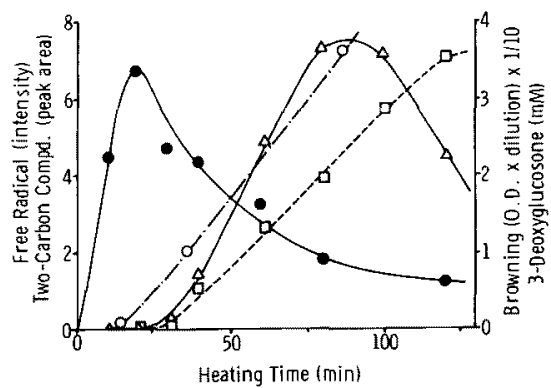

FIG. 5. Formation of Glyoxaldialkylimine, 3Deoxyglucosone and the Free Radical during the Reaction of D-Glucose with $t$-Butylamine $(1 \mathrm{M}$ each) in Ethanol.

- - , glyoxaldialkylimine; --O--, 3-deoxyglucosone; $-\triangle-$, free radical; $--\square--$, browning (absobancy at $420 \mathrm{~nm})$.

free radical species or the appearance of the brown color. This indicated that the formation of the two-carbon fragment occurs independently from that of 3-deoxyglucosone, that the fragmentation probably occurs to glucose or glycosylamine molecule.

One of the possibilities of the mechanism of the fragmentation of sugar molecule is the fission by the reverse-aldol condensation occurring at $\mathrm{C} 2-\mathrm{C} 3$ bond that has been supposed to occur under highly alkaline condition. ${ }^{57}$ If a similar mechanism is operating in the present case of the formation of glyoxaldialkylimines, higher alkalinity may increase the yield of these products. The experiment (Fig. 6B), 


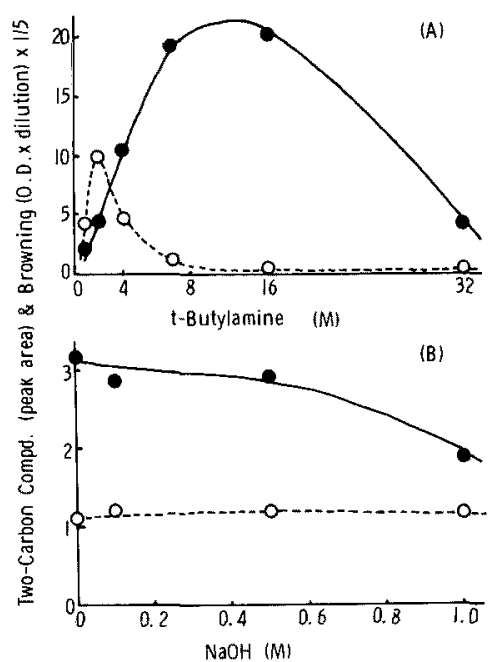

FIG. 6. Changes in the Formation of Glyoxaldialkylimine with Increasing Concentration of Amine (A) or Alkali (B).

(A) reaction of D-glucose (1 M) with different concentration of $t$-butylamine in ethanol refluxed for $40 \mathrm{~min}$; (B) reaction of D-glucose $(1 \mathrm{M})$ with $t$-butylamine $(2 \mathrm{M})$ in ethanol with different concentration of sodium hydroxide refluxed for $40 \mathrm{~min}$.

- - - glyoxaldialkylimine; --- - --., browning (absorbancy at $420 \mathrm{~nm}$ ).

however, showed that addition of sodium hydroxide had the opposite effect of slightly decreasing the yield of the two-carbon compound from glucose by the reaction with $t$ butylamine. However, using a larger excess of the amine to glucose remarkably increased its yield which was maximum at 10 fold excess of the amine to glucose (Fig. 6A). Though circumstantial, this seems to suggest the possibility that the amine is involved in the process of fragmentation, and that the twocarbon fragment probably comes from the aminated sugar, rather than the sugar itself. The evidence, however, is not yet conclusive and the two hypothetical pathways of the formation of glyoxaldialkylimine are presented in Fig. 7. The preference of the Route B over $\mathrm{A}$ is also supported by the result of degradation of glucose in $0.1 \mathrm{M}$ sodium hydroxide, which showed no evidence of the formation of the two-carbon fragment, either glycolaldehyde, glyoxal or glycolic acid.

Further studies of the pathway of the formation of glyoxaldialkylimines is under way, and the result will be published in relation to the formation of the radical species and the browning.

\section{EXPERIMENTAL}

Materials and methods. Authentic specimen of glyoxaldicyclohexylimine $^{6)}$ and 3 -deoxy-D-glucosone ${ }^{7)}$ were prepared according to literatures. Glyoxalbisphenylhydrazone was synthesized by the reaction of glyoxal with phenylhydrazine hydrochloride in aqueous sodium acetate, and its structure was confirmed by ${ }^{1} \mathrm{H}$-NMR. Other reagents were of guaranteed grade.

Analytical and preparative TLC were carried out on precoated Merk silica gel plates and on those coated with Wakogel B $(20 \times 20 \mathrm{~cm})$, respectively, using the same solvent system (ethyl acetate-methanol-chloroform, $5: 2: 1)$. Solution of $2,4-\mathrm{DNP}(3 \%$ in the mixture of ethanol, water and sulfuric acid $(70: 20: 15))$ was used for the visualization and location of the spots and bands.

IR, ${ }^{1} \mathrm{H}-\mathrm{NMR}$ and mass spectra were recorded using JASCO A-3, JNM-MH-100 and JMS-D-100 spectrometer, respectively. GLC analyses of the reduced and

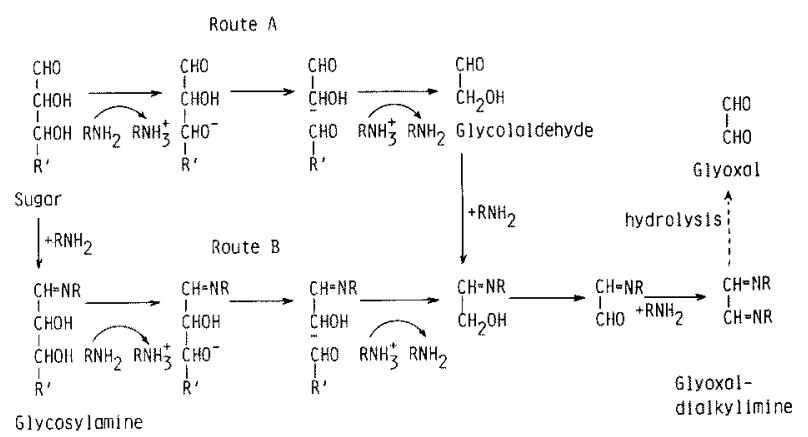

Fig. 7. Possible Pathways on the Formation of Two-Carbon Compound by the Reaction of Sugar with Amine. 
acetylated products were carried out on a Schimadzu GC4BMPF on silicone XE- 60 column at $110^{\circ} \mathrm{C}$ in the case of glyoxal and $180^{\circ} \mathrm{C}$ in the case of 3-deoxyglucosone. Determination of the spot on TLC plate was done by use of a Shimadzu CS-100 TLC scanner with the absorbancy at $400 \mathrm{~nm}$.

Isolation and identification of glyoxal (Fig. 2). D-Glucose and $t$-butylamine $(0.1 \mathrm{~mol}$ each) in $100 \mathrm{ml}$ of $95 \%$ ethanol was refluxed for $40 \mathrm{~min}$, concentrated under vacuum, added with small amount of water and extracted with $50 \mathrm{ml}$ ethyl acetate. The concentrate of the extract was chromatographed on a reversed-phase column (Bondapak C-18), washed with water and eluted by $2: 1$ watermethanol. The concentrated eluate washed with ethyl acetate was developed on silica gel TLC plate ( 5 sheets). The band found positive to 2,4-DNP reagent was scraped off and eluted with methanol, and subsequent evaporation gave white residue (about $1 \mathrm{mg}$ ) (product 1).

In another run the concentrated extract with ethyl acetate in the above procedure was directly developed on TLC plate. Elution of the 2,4-DNP-positive band with methanol followed by concentration gave brown syrup (about $0.3 \mathrm{~g}$ ) (product 2).

a) Reduction and acetylation. The product 1 was treated with $15 \mathrm{mg}$ of sodium borohydride in water overnight. After acidification by addition of Amberite IR$120\left(\mathrm{H}^{+}\right)$and filtration the solution was repeatedly evaporated with repeated additions of methanol. The residue was dissolved in $0.1 \mathrm{ml}$ pyridine and $0.1 \mathrm{ml}$ acetic anhydride and heated at $70^{\circ} \mathrm{C}$ for $2 \mathrm{hr}$. The reaction mixture was analyzed by GLC.

b) Treatment with 2,4-DNP. A few drop of 2,4-DNP reagent was added to a part of product 1 in water. The reaction mixture was extracted with ethyl acetate, and the extract was developed on silica gel TLC using a solvent system of benzene-hexane-ethyl acetate $(5: 2: 1)$.

c) Phenylhydrazone. Aqueous solution $(8 \mathrm{ml})$ of phenylhydrazine hydrochloride $(0.75 \mathrm{~g})$ and sodium acetate $(1.2 \mathrm{~g})$ was added to the product 2 . The yellow precipitate produced immediately after the treatment was dissolved in benzene followed by washing with water. The solution was then chromatographed on a silica gel column. Eluate with benzene gave yellow residue (11.2 $\mathrm{mg}$ ), which was crystallized from ethanol to give yellow plate. mp $170 \sim 171^{\circ} \mathrm{C} .{ }^{1} \mathrm{H}-\mathrm{NMR}$ (DMSO- $d_{6}$ ), $\delta 6.6 \sim 7.2$ multiplet $(10 \mathrm{H}$, aromaticH $), 7.6$ singlet $(2 \mathrm{H}$, alkylidene $\mathrm{H})$ and 10.25 singlet $(2 \mathrm{H}, \mathrm{sec}$-amine $\mathrm{H})$

Effect of acid- or silica gel-treatment on 2,4-DNPpositive product (Fig. 3). The reaction mixture of glucose with $t$-butylamine ( $1 \mathrm{~m}$ each in $95 \%$ ethanol, $20 \mathrm{~min}$ reflux) was prepared. A portion was acidified to $\mathrm{pH} 1(\mathrm{HCl})$ and was kept at $40^{\circ} \mathrm{C}$ for $15 \mathrm{~min}$, cooled and neutralized. Both the treated solution and the remainder of the reaction mixture were extracted with ethyl acetate after addition of water. Each extract was repeatedly washed with water and, each water layer was with ethyl acetate. The combined ethyl acetate extracts and the combined water layers were similarly subjected to TLC (Fig. 3, Nos. 2, 3, 6 and 7 ), after concentration.

Treatment with silica gel was carried out on the TLC plate, on which an aliquot of the reaction mixture was applied. One half of the band was extracted with ethyl acetate and the other half was with water, and the extracts were subjected to TLC (Fig. 3, Nos. 4 and 5).

In Fig. 3 it is observed that all of the spots for the aqueous solution have slightly lower $R f$ values than those from ethyl acetate or ethanol solutions. This difference is probably due to the hydration of glyoxal in water, and does not represent different identities.

Glyoxaldicyclohexylimine from reaction mixture. DGlucose $(0.05 \mathrm{~mol})$ and cyclohexylamine $(0.05 \mathrm{~mol})$ in $50 \mathrm{ml} 95 \%$ ethanol were reacted under reflux for $15 \mathrm{~min}$. Seventy $\mathrm{ml}$ of water was added and the mixture was extracted with $70 \mathrm{ml}$ of chloroform, where most of the brown color was transferred to the solvent phase. The solvent was dried and concentrated to $50 \mathrm{ml}$, added with $5 \mathrm{ml}$ ethyl acetate and stood in a freezer to yield $0.14 \mathrm{~g}$ of white lustrous plates, which was recrystallized from ethyl acetate. $\mathrm{mp} 151 \sim 152^{\circ} \mathrm{C} .{ }^{1} \mathrm{H}-\mathrm{NMR}\left(\mathrm{CDCl}_{3}\right), \delta 1.30 \sim 1.80$ broad multiplet $(20 \mathrm{H}), 3.06$ broad multiplet $(2 \mathrm{H})$ and 7.82 sharp singlet. Mass $m / e$ (relative intensity) 41 (80), 55 (96), $82(100), 83\left(\mathrm{C}_{6} \mathrm{H}_{11}^{+}, 77\right), 95(58), 177\left(\mathrm{M}-\mathrm{C}_{3} \mathrm{H}_{7}, 74\right)$ and $220\left(\mathrm{M}^{+}, 10\right)$. IR, see Fig. 4.

In another run D-glucose $(0.05 \mathrm{~mol})$ and cyclohexylamine $(0.25 \mathrm{~mol})$ in $50 \mathrm{ml} 99 \%$ ethanol were refluxed for $30 \mathrm{~min}$, and the reaction mixture dried under vacuum was extracted three $100 \mathrm{ml}$ portions of hexane. Evaporation of the extract gave $0.35 \mathrm{~g}$ of colorless plates. The aqueous washings of the residue of the extraction was again extracted with hexane, which gave additional $0.47 \mathrm{~g}$ of the product upon concentration. The combined products recrystallized from ethyl acetate melted at $151 \sim 152^{\circ} \mathrm{C}$.

Determination of glyoxaldialkylimines by $T L C$. One $\mu 1$ of the reaction mixture was developed on the TLC plate $(10 \times 10 \mathrm{~cm})$, and sprayed with the 2,4-DNP reagent. After $10 \mathrm{~min}$ an orange spot of glyoxal appeared around $R f 0.7$ was measured densitometrically.

Determination of 3-deoxyglucosone. Two $\mathrm{ml}$ of the reaction mixture diluted 20 times with water was treated with $15 \mathrm{mg}$ sodium borohydride overnight. The reduced product was acetylated as was described in an earlier section for glyoxal and similarly analyzed by GLC. The amount of 3-deoxyglucosone was correlated with the peak area obtained.

Acknowledgment. The authors thank Dr. K. Tsuji of the Institute of Physical and Chemical Research for helpful advice and Miss $\mathrm{H}$. Kato for technical assistance. 


\section{REFERENCES}

1) M. Namiki and T. Hayashi, J. Agric. Food Chem., 23, 487 (1975); T. Hayashi, T. Ohta and M. Namiki, ibid., 25, 1282 (1977).

2) J. E. Hodge, ibid., 1, 928 (1953).

3) H. Budzikiewicz, C. Djerassi and D. H. Williams,
"Mass Spectrometry of Organic Compounds," Holden-Day, Inc., London, 1967, p. 304.

4) H. Kato, Bull. Agric. Chem. Soc. Jpn., 24, 1 (1960).

5) J. F. Harris, Carbohyd. Res., 23, 207 (1972).

6) J. F. Carson, J. Am. Chem. Soc, 75, 4337 (1953).

7) H. El Khadem, D. Horton, M. Meshreki and M. A. Nashed, Carbohyd. Res., 17, 183 (1971). 